\title{
ON THE FREDHOLM ALTERNATIVE FOR NONLINEAR FUNCTIONAL EQUATIONS IN BANACH SPACES
}

\author{
PETER HESS ${ }^{1}$
}

\begin{abstract}
The well-known Fredholm alternative theorem for compact linear operators is carried over to a class of noncompact, asymptotically linear mappings of monotone type of a real reflexive Banach space into its dual. An application to a nonlinear elliptic boundary value problem is given.
\end{abstract}

Introduction. Let $X$ be a Banach space, $X^{*}$ its conjugate space, and let $(w, u)$ denote the value of the functional $w^{*} \in X^{*}$ at $u \in X$. For $C$ a compact linear operator of $X$ into itself, $C^{*}: X^{*} \rightarrow X^{*}$ its adjoint operator, the following ("Fredholm alternative") is well known:

(i) The dimensions of the nullspaces $K(I-C)$ and $K\left(I-C^{*}\right)$ are finite and equal;

(ii) the equation $(I-C) u=f$ is solvable for given $f \in X$ if and only if $f \in K\left(I-C^{*}\right)-$.

Here $K\left(I-C^{*}\right)=\left\{u \in X:(w, u)=0\right.$ for $\left.w \in K\left(I-C^{*}\right)\right\}$.

In the recent paper [5] Kačurovskil carries over this Fredholm theory to nonlinear equations of the form $(I-D) u=f$ with $D$ compact and asymptotically linear, and gives applications to integral equations of Hammerstein and Urysohn type as well as to ordinary differential equations. His result is in general however not applicable to nonlinear elliptic boundary value problems.

It is our purpose in the present note to prove an extension of Kačurovskiǐ's resuit to the class of mappings of type $(S)$ of a real reflexive Banach space $X$ into $X^{*}$, which admits applications to boundary value problems. Mappings of type $(S)$ have grown increasingly important in the study of elliptic equations and solvability criterions involving homotopy arguments (cf. [1], [3], [4]). We use the symbols " $\rightarrow$ " and " $\rightarrow$ " to denote strong and weak convergence, respectively.

Received by the editors March 29, 1971 and, in revised form, August 2, 1971.

AMS 1969 subject classifications. Primary 4780; Secondary 4610, 3545.

Key words and phrases. Nonlinear mapping, mapping of monotone type, real reflexive Banach space, asymptotically linear operator, Fredholm alternative, linear Fredhoim operator, nonlinear elliptic boundary value problem.

1 Results obtained at the University of Chicago; research supported by the Schweizerischer Nationalfonds.

(c) American Mathematical Society 1972 
Definition. $A$ (nonlinear) mapping $A: X \rightarrow X^{*}$ is of type $(S)$ provided $u_{n} \rightarrow u$ in $X$ and $\lim \left(A u_{n}-A u, u_{n}-u\right)=0$ imply that $u_{n} \rightarrow u$.

The mapping $A: X \rightarrow X^{*}$ is further said to be demicontinuous if it is continuous from the strong topology on $X$ to the weak topology on $X^{*}$, and bounded if it maps bounded sets onto bounded sets.

In $\S 1$ we investigate the class of bounded linear operators of type $(S)$. We show

THEOREM 1. Let $X$ be a real reflexive Banach space and $T$ a bounded linear mapping of $X$ into $X^{*}$ of type $(S)$. Then $T$ is a Fredholm operator with index 0 , i.e. the range $R(T)$ is closed in $X^{*}$ and

$$
\operatorname{dim} K(T)=\operatorname{dim} K\left(T^{*}\right)<\infty
$$

$\S 2$ embodies the proof of our main result,

THEOREM 2. Let $X$ be a real reflexive Banach space and $A: X \rightarrow X^{*} a$ bounded demicontinuous mapping of type $(S)$. Suppose $A$ admits the decomposition $A=T+N$, where $T$ is a bounded linear operator of type $(S)$ and $N$ a mapping with $\lim _{\|u\| \rightarrow \infty}\|u\|^{-1}\|N u\|=0$. Suppose in addition that $R(N) \subset K\left(T^{*}\right)^{\perp}$. In order that the functional equation $A u=f$ is solvable for a given $f \in X^{*}$ it is necessary and sufficient that $f \in K\left(T^{*}\right)^{\perp}$.

$\S 3$ finally brings an application of Theorem 2 to a nonlinear elliptic boundary value problem.

Our considerations are essentially based on recent results by the author on nonlinear, asymptotically homogeneous and odd mappings of monotone type (cf. [3], [4]).

1. We start our detailed discussion with the proof of Theorem 1. This is the content of the following three lemmas.

LemMa 1. Let $T$ be a bounded linear operator of type $(S)$. Then its range $R(T)$ is closed in $X^{*}$.

Proof. By a known result [2, Theorem VI.6.5], it suffices to show that whenever $C$ is a bounded closed subset of $X$, then the set $T(C)$ is closed in $X^{*}$. Let $\left\{u_{n}\right\}$ be a sequence in $C$ with $T u_{n} \rightarrow w$. As a consequence of the boundedness of $\left\{u_{n}\right\}$ and the reflexivity of $X$, we may extract an infinite subsequence such that $u_{n} \rightarrow u$. Since $\lim \left(T\left(u_{n}-u\right), u_{n}-u\right)=0$, property $(S)$ implies that $u_{n} \rightarrow u$. It follows that $T u_{n} \rightarrow T u=w$ and, since $C$ is closed, that $w \in T(C)$. Q.E.D.

LEMMA 2. A bounded linear operator $T$ of type $(S)$ has finite-dimensional nullspace $K(T)$. 
Proof. Under the assumption that $\operatorname{dim} K(T)=+\infty$, we construct an infinite sequence $\left\{u_{n}\right\}$ in $K(T)$ with $\left\|u_{n}\right\|=1$ and

$$
\left\|u_{n}-u_{m}\right\|_{i} \geqq \frac{1}{2}
$$

for all $n$ and $m \neq n$. We may pass to an infinite subsequence with $u_{n} \rightarrow u$. Since $T u_{n}=0$ for all $n, \lim \left(T\left(u_{n}-u\right), u_{n}-u\right)=0$. It follows from property $(S)$ of the mapping $T$ that $\left\{u_{n}\right\}$ is strongly convergent, which is impossible because of (1). Q.E.D.

Since the space $X$ is assumed to be reflexive, the linear operator $T^{*}$ adjoint to $T$ can be considered as a mapping of $X$ into $X^{*}$. We observe that $T$ is of type $(S)$ if and only if $T^{*}$ is of type $(S)$. For the proof of the next lemma we need the following result (cf. [3], [4]):

Proposition. Let $A$ be a (nonlinear) bounded demicontinuous mapping of the real reflexive Banach space $X$ into $X^{*}$. Suppose that $A$ is of type $(S)$ and admits a decomposition $A=T+N$, with $T$ a bounded linear operator of type $(S)$ and $N$ a mapping satisfying $\lim _{\|u\| \rightarrow \infty}\|u\|^{-1}\|N u\|=0$. If $T u=0$ only for $u=0$, then $A$ is surjective.

LemMa 3. If $T$ is a continuous linear operator of type $(S)$, then $\operatorname{dim} K(T)=\operatorname{dim} K\left(T^{*}\right)$.

Proof. By symmetry of the problem, it suffices to consider the case $n^{*}=\operatorname{dim} K\left(T^{*}\right) \geqq \operatorname{dim} K(T)=n$. Let $\left\{u_{1}, \cdots, u_{n}\right\}$ be a basis of the subspace $K(T)$. There exist elements $u_{1}^{*}, \cdots, u_{n}^{*}$ in $X^{*}$ such that $\left(u_{i}^{*}, u_{j}\right)=\delta_{i j}$ (cf. [6, p. 151]). Let further $\left\{v_{1}, \cdots, v_{n}\right\}$ be iinearly independent elements of $K\left(T^{*}\right)$ and $v_{1}^{*}, \cdots, v_{n}^{*}$ functionals in $X^{*}$ with $\left(v_{i}^{*}, v_{j}\right)=\delta_{i j}$.

We define a new linear mapping $T^{\prime}: X \rightarrow X^{*}$ by

$$
T^{\prime} u=T u+\sum_{k=1}^{n}\left(u_{k}^{*}, u\right) v_{k}^{*} \quad(u \in X) .
$$

The operator $T^{\prime}$ is again of type $(S)$. We further claim that $K\left(T^{\prime}\right)=\{0\}$. Indeed, let $u \in X$ be such that $T^{\prime} u=0$. For $1 \leqq j \leqq n$,

$$
0=\left(T^{\prime} u, v_{j}\right)=\left(T u, v_{j}\right)+\sum_{k=1}^{n}\left(u_{k}^{*}, u\right)\left(v_{k}^{*}, v_{j}\right)=0+\left(u_{j}^{*}, u\right) .
$$

Hence

$$
0=T^{\prime} u=T u+0,
$$

i.e. $u \in K(T)$. Therefore $u$ admits a representation $u=\sum_{k=1}^{n} \alpha_{k} u_{k}$. The relation $0=\left(u_{j}^{*}, u\right)=\alpha_{j}$ for $1 \leqq j \leqq n$ implies that $u=0$.

Assume now that $n^{*}>n$. Then there exists $v \in K\left(T^{*}\right), v \neq 0$, such that $\left(v_{k}^{*}, v\right)=0$ for $1 \leqq k \leqq n$. Since $K\left(T^{\prime}\right)=\{0\}$, it follows from the Proposition 
that $R\left(T^{\prime}\right)=X^{*}$. Let $J$ denote the duality mapping of $X$ into the set $2^{X}$ of all subsets of $X^{*}$,

$$
J u=\left\{q \in X^{*}:(q, u)=\|q\|\|u\|,\|q\|=\|u\|\right\} .
$$

By the Hahn-Banach theorem, $J u \neq \varnothing$ for all $u \in X$. Let $q_{0} \in J v$. Since $R\left(T^{\prime}\right)=X^{*}$, there exists an element $u_{0} \in X$ with $T^{\prime} u_{0}=q_{0}$. We infer that

$$
\begin{aligned}
\|v\|^{2} & =\left(q_{0}, v\right)=\left(T^{\prime} u_{0}, v\right) \\
& =\left(T u_{0}, v\right)+\sum_{k=1}^{n}\left(u_{k}^{*}, u_{0}\right)\left(v_{k}^{*}, v\right)=0,
\end{aligned}
$$

in contradiction to $v \neq 0$. Q.E.D.

REMARK. In the case that $X=X^{*}=H$ is a Hilbert space, Lemma 3 can be proved considerably simpler by observing that there exists an exponent $r_{0}$ with the property that $K\left(T^{r}\right)=K\left(T^{r_{0}}\right)$ for $r \geqq r_{0}$. No reference to the Proposition is then needed.

2. We proceed to the

Proof OF Theorem 2. It is trivial by the assumption on the range of $N$ that the condition is necessary. For the proof of the sufficiency, suppose $f \in K\left(T^{*}\right)^{\perp}$. Let $n=\operatorname{dim} K(T)=\operatorname{dim} K^{\prime}\left(T^{*}\right)$, and define the linear operator $T^{\prime}$ :

$$
T^{\prime} u=T u+\sum_{k=1}^{n}\left(u_{k}^{*}, u\right) v_{k}^{*}
$$

as in the proof of Lemma 3. Then $T^{\prime}$ is of type $(S)$ and $K\left(T^{\prime}\right)=\{0\}$. Let further $A^{\prime}=T^{\prime}+N$. By the Proposition, $R\left(A^{\prime}\right)=X^{*}$. Consequently there exists $u_{0} \in X$ with $A^{\prime} u_{0}=f$. We infer that

$$
\sum_{k=1}^{n}\left(u_{k}^{*}, u_{0}\right) v_{k}^{*}=f-T u_{0}-N u_{0}
$$

where the right side is an element of $R(T)$. Set $\alpha_{k}=\left(u_{k}^{*}, u_{0}\right)$ and suppose $\sum_{k=1}^{n} \alpha_{k} v_{k}^{*}=T u$ for some $u \in X$. Since $\alpha_{j}=\left(T u, v_{j}\right)=\left(u, T^{*} v_{j}\right)=0$ for $1 \leqq j \leqq n$, it follows that $\sum_{k=1}^{n} \alpha_{k} v_{k}^{*}=0$ and hence $f-T u_{0}-N u_{0}=f-A u_{0}=0$. Q.E.D.

3. We now apply Theorem 2 to the discussion of the solvability of the generalized Dirichlet problem for an asymptotically linear elliptic equation of the form

$$
(\mathscr{A} u)(x)=-\sum_{i, j=1}^{n} \frac{\partial}{\partial x_{i}}\left(a_{i j}(x) \frac{\partial u}{\partial x_{j}}\right)+a_{0}\left(x, u, \frac{\partial u}{\partial x_{1}}, \cdots, \frac{\partial u}{\partial x_{n}}\right)=f(x),
$$

defined on an open, bounded, smoothly bounded subset $\Omega$ of a Euclidean 
space $R^{n}$. The generic point is denoted by $x=\left(x_{1}, \cdots, x_{n}\right)$. We impose the following conditions on the operator $\mathscr{A}$ (all functions are assumed to be real-valued):

(I) $a_{i j} \in L^{\infty}(\Omega) \quad(1 \leqq i, j \leqq n)$, and $\sum_{i, j=1}^{n} a_{i j}(x) \xi_{i} \xi_{j} \geqq c|\xi|^{2} \quad(c>0)$, for $\xi=\left(\xi_{1}, \cdots, \xi_{n}\right) \in R^{n}$ and a.a. $x \in \Omega$.

(II) The function $a_{0}\left(x, \xi_{0}, \xi_{1}, \cdots, \xi_{n}\right)$ is measurable in $x$, continuous in $\xi=\left(\xi_{0}, \xi_{1}, \cdots, \xi_{n}\right) \in R^{n+1}$, and asymptotically linear in the following sense: there exist functions $b_{i} \in L^{\infty}(\Omega)(i=0, \cdots, n)$ such that

$$
\left|\frac{1}{\lambda} a_{0}(x, \lambda \hat{\xi})-\sum_{i=0}^{n} b_{i}(x) \xi_{i}\right| \leqq c(\lambda)(|\hat{\xi}|+1),
$$

with $c(\lambda) \rightarrow 0$ as $\lambda \rightarrow+\infty$.

For $u \in L^{2}(\Omega)$, let $\|u\|_{0}=\left(\int_{\Omega}|u|^{2} d x\right)^{1 / 2}$, and let $X=W_{0}^{1,2}(\Omega)$ denote the completion of $\mathscr{D}(\Omega)$ with respect to the norm

$$
\|u\|_{1}=\left(\|u\|_{0}^{2}+\sum_{i=1}^{n}\left\|\frac{\partial u}{\partial x_{i}}\right\|_{0}^{2}\right)^{1 / 2} .
$$

The space $X$ is a Hilbert space provided with the inner product

$$
(u, v)_{1}=\int_{\Omega}\left(u v+\sum_{i=1}^{n} \frac{\partial u}{\partial x_{i}} \frac{\partial v}{\partial x_{i}}\right) d x .
$$

The conditions (I) and (II) imply that the (nonlinear) Dirichlet form

$$
a(u, v)=\sum_{i, j=1}^{n} \int_{\Omega} a_{i j}(x) \frac{\partial u}{\partial x_{j}} \frac{\partial v}{\partial x_{i}} d x+\int_{\Omega} a_{0}\left(x, u, \frac{\partial u}{\partial x_{1}}, \cdots, \frac{\partial u}{\partial x_{n}}\right) v d x
$$

corresponding to the differential operator $\mathscr{A}$ is defined for all $u, v$ in $X$.

DEFINITION. A function $u$ is said to be a solution of the generalized Dirichlet problem for the equation $\mathscr{A} u=f, f \in L^{2}(\Omega)$ given, if

(i) $u \in X$;

(ii) $a(u, v)=\int_{\Omega} f v d x$ for all $v \in X$.

Let $\mathscr{T}$,

$$
\mathscr{T} u=-\sum_{i, j=1}^{n} \frac{\partial}{\partial x_{i}}\left(a_{i j}(x) \frac{\partial u}{\partial x_{j}}\right)+\sum_{i=1}^{n} b_{i}(x) \frac{\partial u}{\partial x_{i}}+b_{0}(x) u,
$$

denote the (linear) asymptotic part of $\mathscr{A}$ and $\mathscr{T}^{\prime}$ the differential expression formally adjoint to $\mathscr{T}$.

THEOREM 3. Let the functions $v_{1}, \cdots, v_{m}$ form a basis for the subspace (of $X$ ) consisting of solutions of the generalized Dirichlet problem for the equation $\mathscr{T}^{\prime} v=0$. Suppose in addition that $a\left(u, v_{k}\right)=0$ for all $u \in X$ and $k=1, \cdots, m$. Then the generalized Dirichlet problem for the equation 
$\mathscr{A} u=f$ is solvable for given $f \in L^{2}(\Omega)$ if and only if $\int_{\Omega} f v_{k} d x=0$ for $k=$ $1, \cdots, m$.

Proof. As a consequence of conditions (I) and (II) there exists a bounded continuous operator $A$ in $X$ such that $a(u, v)=(A u, v)_{1}$ for $u, v$ in $X$ (cf. [1]). Similarly, let $t(u, v)$ be a bilinear form associated with $\mathscr{T}$, and $T$ the bounded linear operator in $X$ induced by $\mathscr{T}$. By the uniform ellipticity of $\mathscr{T}$, a Gårding inequality

$$
t(u, u) \geqq c_{1}\|u\|_{1}^{2}-c_{2}\|u\|_{0}^{2} \quad\left(c_{1}>0\right)
$$

is satisfied for $u \in X$. Since the imbedding of $X$ in $L^{2}(\Omega)$ is compact, we infer that the operators $A$ and $T$ are of type $(S)$. Moreover

$$
\lim _{\|u\|_{1} \rightarrow \infty}\|u\|_{1}^{-1}\|(A-T) u\|_{1}=0 .
$$

To given $f \in L^{2}(\Omega)$ there exists $g_{f} \in X$ such that $\int_{\Omega} f v d x=\left(g_{f}, v\right)_{1}$ for $v \in X$. Necessary and sufficient for a function $u \in X$ to be solution of the generalized Dirichlet problem for the equation $\mathscr{A} u=f$ is that $(A u, v)_{1}=$ $a(u, v)=\int_{\Omega} f v d x=\left(g_{f}, v\right)_{1}$ for $v \in X$, i.e. that $A u=g_{f}$. It is readily seen that the functions $v_{1}, \cdots, v_{m}$ form a basis of $K\left(T^{*}\right)$. Further $R(A-T) \subset$ $K\left(T^{*}\right)^{\perp}$. The assertions of Theorem 3 then follow immediately from Theorem 2.

EXAMPLE. Let $\Omega=\left\{x \in R^{2}:|x|<1\right\}$ and

$$
(\mathscr{A} u)(x)=-\Delta u-\mu u+(1+|u(x)|+|u(-x)|)^{-1}(u(x)-u(-x)) .
$$

It is known that $\mathscr{T}=\mathscr{T}^{\prime}=-\Delta-\mu$ (with Dirichlet boundary conditions) has nontrivial nullspace iff $\mu=k_{n, m}^{2}(n=0,1, \cdots ; m=1,2, \cdots)$, where $k_{n, m}$ denotes the $m$ th positive root of the Bessel function $J_{n}$. Writing $x$ in polar coordinates $(r, \vartheta)$, the corresponding eigenfunctions have the form

$$
v=J_{n}\left(k_{n, m} r\right)\left\{\begin{array}{c}
\cos n \vartheta \\
\sin n \vartheta
\end{array}\right\}
$$

Since the (asymptotically vanishing) nonlinear part of $\mathscr{A}$ is odd in $x \in \Omega$, the additional assumption in Theorem 3 is satisfied if $\mu=k_{n, m}^{2}$ with $n$ even. (Remark: The nonlinear part of $\mathscr{\alpha}$ is not exactly of the form introduced in condition (II), but one may consider it as a function of the two variables $\xi_{0}=u(x), \xi_{0}=u(-x)$.)

\section{REFERENCES}

1. F. E. Browder, Existence theorems for nonlinear partial differential equations, Proc. Sympos. Pure Math., vol. 16, Amer. Math. Soc., Providence, R.I., 1970, pp. 1-60. 
2. N. Dunford and J. T. Schwartz, Linear operators. I: General theory, Pure and Appl. Math., vol. 7, Interscience, New York and London, 1958. MR 22 \#8302.

3. P. Hess, Nonlinear functionai equations in Banach spaces and homotopy arguments, Bull. Amer. Math. Soc. 77 (1971), 211-215.

4. ._ On nonlinear mappings of monotone type homotopic to odd operators, J. Functional Analysis (to appear).

5. R. I. Kačurovskiǐ, On Fredholm theory for nonlinear operator equations, Dokl. Akad. Nauk SSSR 192 (1970), 969-972=Soviet Math. Dokl. 11 (1970), 751-754. MR 42 \#911.

6. A. C. Zaanen, Linear analysis. Measure and integral, Banach and Hilbert space, linear integral equations, Interscience, New York; North-Holland, Amsterdam; Noordhoff, Groningen, 1953. MR 15, 878.

Department of Mathematics, University of California, Berkeley, California 94720 